13

\title{
Теоретическое и экспериментальное исследование миниатюрной планарной замедляющей системы на диэлектрической подложке для лампы бегущей волны $W$-диапазона
}

\author{
(C) Р.А. Торгашов, ${ }^{1,2}$ Н.М. Рыскин, ${ }^{1,2}$ А.Г. Рожнев, ${ }^{1,2}$ А.В. Стародубов, ${ }^{1,2}$ А.А. Сердобинцев, ${ }^{2}$ А.М. Павлов, ${ }^{2}$ \\ В.В. Галушка, ${ }^{2}$ И.Ш. Бахтеев, ${ }^{3}$ С.Ю. Молчанов ${ }^{4}$
}

${ }^{1}$ Саратовский фрилиал Института радиотехники и электроники им. В.А. Котельникова РАН, 410019 Саратов, Россия

${ }^{2}$ Саратовский национальный исследовательский государственный университет им. Н.Г. Чернышевского, 410012 Саратов, Россия

${ }^{3} \mathrm{AO}$ „Центральный научно-исследовательский институт измерительной аппаратуры“, 410012 Саратов, Россия

${ }^{4}$ Институт фоизики твердого тела РАН, 142432 Черноголовка, Россия e-mail: torgashovra@gmail.com

Поступило в Редакцию 2 августа 2019 г.

В окончательной редакции 2 августа 2019 г.

Принято к публикации 20 октября 2019 г.

\begin{abstract}
Представлены результаты исследования миниатюрной планарной замедляющей системы (3С) типа меандр для лампы бегущей волны $W$-диапазона. Проведено компьютерное моделирование электродинамических параметров 3С. Описана новая технология изготовления планарных микрополосковых ЗС с помощью лазерной абляции. Проведено экспериментальное исследование $S$-параметров изготовленных образцов 3С. Полученные результаты хорошо согласуются с результатами трехмерного компьютерного моделирования. Проведены расчеты выходных характеристик лампы бегущей волны с ленточным электронным пучком и планарной 3С.
\end{abstract}

Ключевые слова: лампа бегущей волны, меандр, замедляющая система, диэлектрическая подложка.

DOI: 10.21883/JTF.2020.04.49096.294-19

\section{Введение}

Разработка и создание миниатюрных электровакуумных приборов когерентного электромагнитного излучения короткой части миллиметрового диапазона длин волн является актуальной и перспективной задачей современной СВЧ-электроники [1,2]. Подобные приборы могут найти применение в различных областях науки и техники, в первую очередь, в современных системах беспроводной высокоскоростной передачи данных. Наибольший интерес представляют широкополосные усилители типа лампы бегущей волны (ЛБВ) с выходной мощностью порядка десятков W. C целью повышения мощности и КПД таких устройств целесообразно использование пространственно-развитых замедляющих систем (ЗС) и электронных пучков (ЭП) с большим поперечным сечением, в частности, ленточных. В частности, перспективными представляются планарные 3С на диэлектрических подложках [3-9]. Они обладают большим замедлением, что позволяет снизить рабочие напряжения, сократить продольные размеры и массу прибора.

В работах [10-12] нами были разработаны планарные 3С типа встречные штыри и меандр на диэлектрических подложках из кварца в $V$-диапазоне $(50-70 \mathrm{GHz})$, проведены теоретическое и экспериментальное исследования их электродинамических параметров. В настоящей работе исследуется возможность разработки подобных ЗС более высокочастотного W-диапазона $(75-110 \mathrm{GHz})$.

\section{1. Моделирование электродинамических параметров замедляющих систем}

На рис. 1 представлено схематическое изображение 3С типа меандр на диэлектрической подложке. Замедляющая система помещена в прямоугольный волновод стандартного сечения WR-10. Размеры структуры указаны в таблице. Предполагается, что замедленная электромагнитная волна в такой системе взаимодействует с ленточным электронным пучком, летящим на расстоянии $a$ от поверхности металла.

Моделирование электродинамических параметров замедляющей системы проводилось в программном пакете COMSOL Multiphysics [13]. Рассматривается один период 3С, а на границы системы вдоль продольного направления накладываются периодические граничные условия Флоке. В качестве материала для подложки выбран кварц, диэлектрическая проницаемость составляет $\varepsilon=3.75$. 
Геометрические размеры 3С

\begin{tabular}{l|c}
\hline \multicolumn{1}{c|}{ Геометрический параметр } & Значение \\
\hline Период, $d, \mu \mathrm{m}$ & 130 \\
Ширина меандра, $l, \mu \mathrm{m}$ & 450 \\
Ширина полоска, $w, \mu \mathrm{m}$ & 32.5 \\
Толщина металлического слоя, $t, \mu \mathrm{m}$ & 5 \\
Толщина подложки, $h, \mu \mathrm{m}$ & 500 \\
Размеры волновода, $\mu \mathrm{m}^{2}$ & $2.4 \times 1.2$
\end{tabular}

На рис. 2, а представлена дисперсионная характеристика 3С. Видно, что она состоит из двух симметричных ветвей, которые смыкаются на $\pi$-виде колебаний. Этот факт объясняется наличием в системе скользящей плоскости симметрии (см., например, $[14,15])$. Для использования в ЛБВ-усилителе необходимо использовать ту ветвь характеристики, которая обладает нормальной дисперсией.

Данная 3С обладает большим замедлением $\left(n=c / v_{p h}=5-10\right)$. Такие значения замедления соответствуют рабочим напряжениям пучка порядка 4-8kV. Зависимость напряжения синхронизма от частоты представлена на рис. $2, b$. Штриховой линей на рис. 2, а показана дисперсионная характеристика пучка при напряжении $5 \mathrm{kV}$.

На рис. 2, с приведена частотная зависимость сопротивления связи для рабочей пространственной гармоники. Оно усреднялось по поперечному сечению пучка $430 \times 50 \mu \mathrm{m}$. При этом считалось, что пучок летит на расстоянии $a=75 \mu \mathrm{m}$ от поверхности ЗС. Сопротивление связи превышает $20 \Omega$ на длинноволновом конце диапазона и убывает с ростом частоты. Для обратной гармоники сопротивление связи принимает значения на 1-2 порядка меньше (cp. [15]), поэтому при дальнейшем моделировании выходных характеристик ЛБВ (см. разд. 4) учитывалась только прямая гармоника.

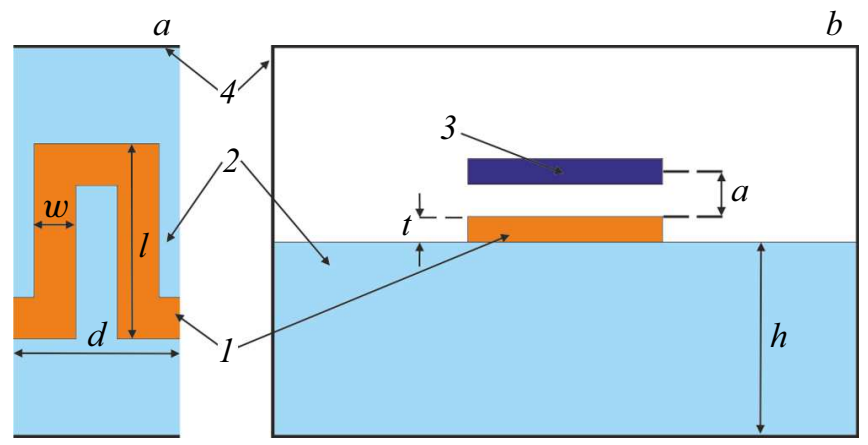

Pис. 1. Схематическое изображение ЗС типа меандр на диэлектрической подложке: $a-$ вид сверху, $b-$ вид сбоку; 1 - меандр, 2 - подложка, 3 - электронный пучок, 4 волновод.
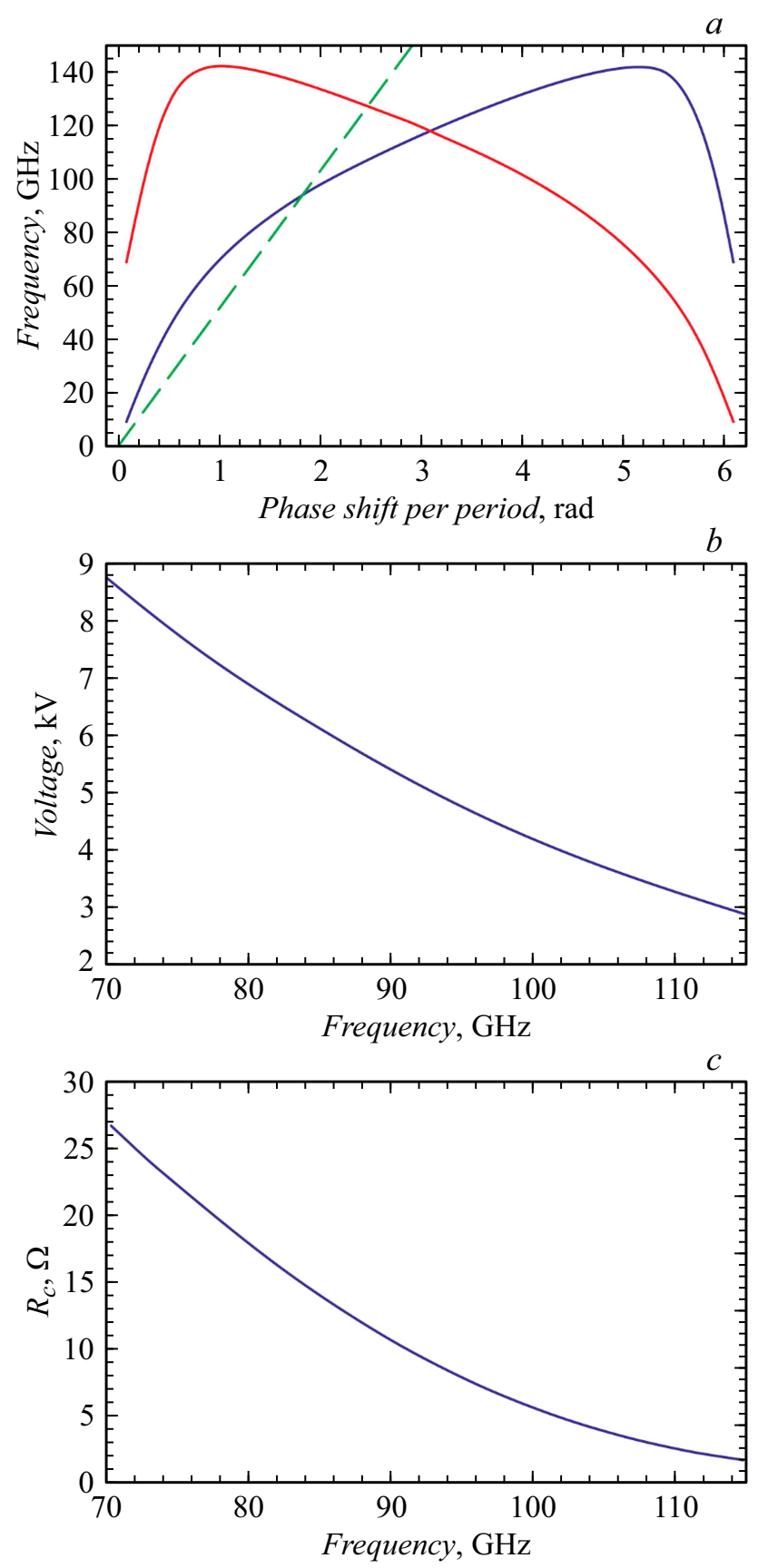

Рис. 2. Электродинамические параметры ЗС $W$-диапазона: $a$ - дисперсионная характеристика (штриховой линией показана дисперсионная характеристика пучка при напряжении $5 \mathrm{kV}) ; b-$ зависимость напряжения синхронизма от частоты; $c$ - зависимость усредненного сопротивления связи от частоты.

\section{2. Технология изготовления}

Ключевой проблемой создания устройств коротковолнового диапазона является изготовление электродинамических структур. С ростом частоты геометрические параметры структур уменьшаются пропорционально рабочей длине волны и могут достигать микронных раз- 


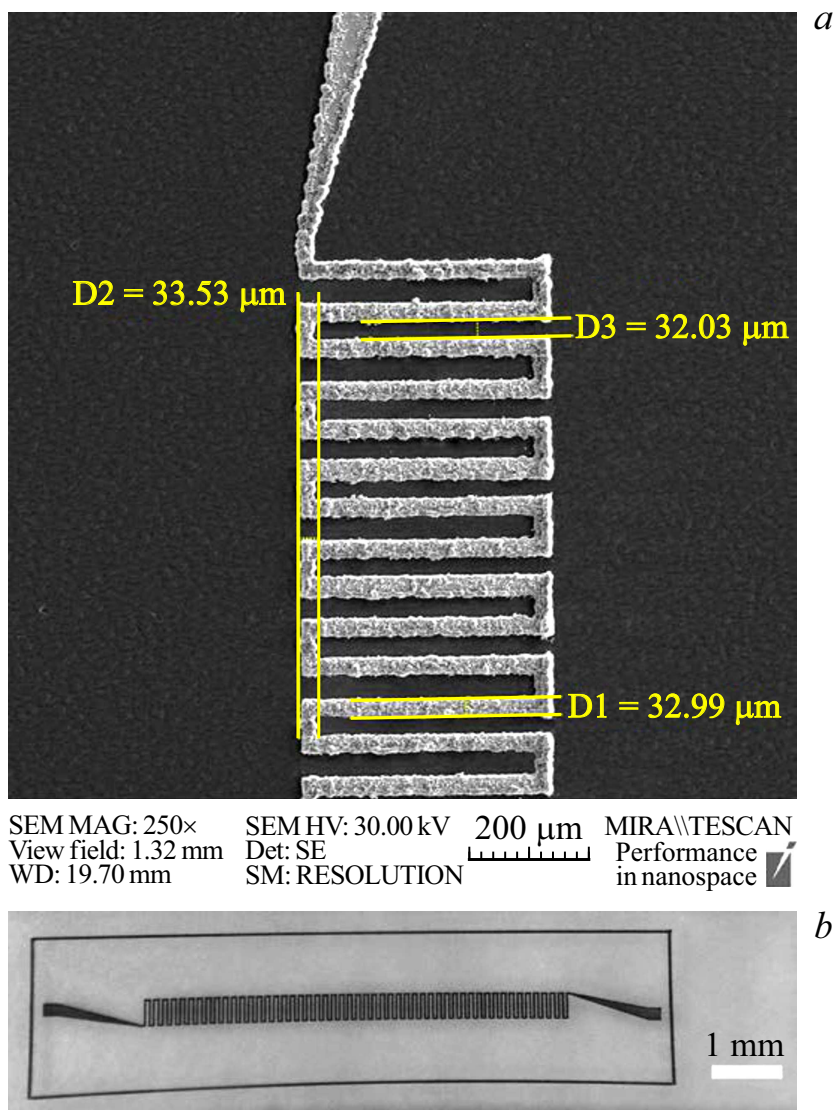

Рис. 3. $a-$ изображение части изготовленной замедляющей системы, полученное со сканирующего электронного микроскопа; $b-$ фотография изготовленной замедляющей системы с оптического микроскопа.

меров. Для изготовления миниатюрных ЗС используются технологии, основанные на фотолитографии и ее разновидностях (LIGA, UV-LIGA), глубокое реактивное ионное травления (DRIE), методы 3D-печати, микро- и нанофрезерование с компьютерным управлением (см., например, [2]). Указанные технологии позволяют создавать микроразмерные структуры с прецизионной точностью, однако высокая стоимость и/или чрезвычайная технологическая трудоемкость изготовления структур с их помощью пока не позволяет говорить о переходе даже к мелкосерийному производству.

Авторами настоящей работы предложена оригинальная технология изготовления планарных ЗС на диэлектрических подложках, которая основана на методах магнетронного напыления и лазерной абляции и включает в себя три основных этапа [16,17]. На первом этапе на кварцевую диэлектрическую подложку наносится проводящий слой толщиной порядка $5 \mu \mathrm{m}$ с помощью технологии магнетронного напыления с использованием мишени из бескислородной меди (марка МБЧ, чистота 99.997\%). Толщина напыляемой пленки контролировалась с помощью методов электронной сканирующей микроскопии и профилометрических измерений.
На следующем этапе происходит формирование рельефа ЗС с помощью лазерной абляции согласно заданной геометрии. Для этого используется коммерчески доступная установка прецизионной лазерной обработки „МиниМаркер-2“, оснащенная иттербиевым импульсным волоконным лазером (длина волны лазерного излучения $1064 \mathrm{~nm}$ ). Выходной пучок со сверхгауссовым распределением мощности фокусировался в пятно диаметром $25 \mu \mathrm{m}$ на медную поверхность. Параметры лазерной абляции для изготовления ЗС были следующими: длительность одиночного импульса составляла $100 \mathrm{~ns}$, частота повторения импульсов составляла $20 \mathrm{kHz}$, скорость движения сканирующего лазерного луча варьировалась в диапазоне 50-200 mm/s, в тангенциальном направлении разрешение составляло 200 линий на $1 \mathrm{~mm}$, что приводило к перекрытию лазерных импульсов на $20 \%$.

На заключительном этапе производится нарезка подложки на отдельные образцы ЗС заданного размера. Для нарезки используется прецизионный алмазный скрайбер RV-129 (ATV Technologie GmbH) с ручным позиционированием.

На рис. 3, a представлена фотография 3С со сканирующего электронного микроскопа MIRA II Tescan. Сформированная медная пленка однородна по толщине, значение которой составляет порядка $5 \mu \mathrm{m}$. Отсутствие сколов и других дефектов подтверждает хорошую адгезию. Изображение с оптического микроскопа представлено на рис. 3, $b$. Геометрические размеры были также подтверждены измерениями с использованием профилометра Dektak® 150 Surface Profiler (Veeco).

Преимуществами разработанной и используемой технологии являются: высокая скорость производства, низкая стоимость по сравнению с технологией фотолитографии, которая использовалась авторами ранее [10,11], а также гибкость технологии в плане быстрого внесения изменений в геометрию ЗС. Полный цикл изготовления может занимать менее одного рабочего дня.

\section{3. Экспериментальное исследование и компьютерное моделирование $S$-параметров}

Экспериментальное исследование $S$-параметров изготовленных макетов было проведено с использованием векторного анализатора цепей ZVA40 (Rohde\&Schwarz) и двух преобразователей частоты ZVA-Z110 (Rohde\&Schwarz), которые преобразуют частотный диапазон анализатора в диапазон 75-110 GHz. Изготовленный образец ЗС длиной $1 \mathrm{~cm}$ (77 периодов 3С) помещался в прямоугольный волновод, соответствующий стандарту WR-10, который с помощью преобразователей частоты подсоединялся к векторному анализатору цепей.

Результаты измерений представлены на рис. 4. В частотном диапазоне 70-110 GHz ослабление не превышает $-10 \mathrm{~dB}$, а коэффициент отражения составляет не 


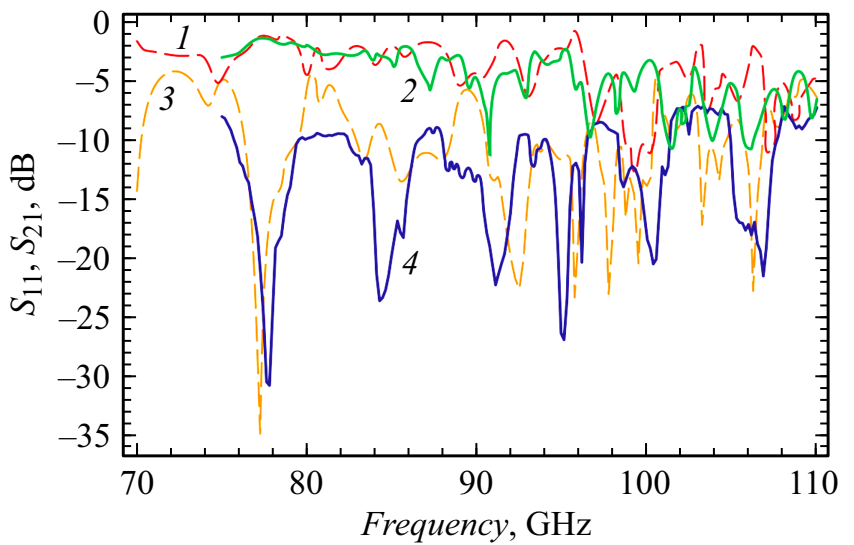

Рис. 4. Частотные зависимости $S$-параметров: $1,2-$ коэффициент затухания S21, 3,4 - коэффициент отражения S11. Штрих - численное моделирование, сплошные линии эксперимент.

более $-5 \mathrm{~dB}$. Подобные характеристики представляются приемлемыми для использования в ЛБВ W-диапазона.

Также было проведено сопоставление экспериментальных результатов с результатами моделирования $S$-параметров 3С. Для этого в программном пакете COMSOL Multiphysics была построена полноразмерная модель ЗС длиной $1 \mathrm{~cm}$ с устройствами ввода/вывода (рис. $3, b$ ), помещенная в прямоугольный волновод. Полагалось, что в волновод подается гармонический входной сигнал, имеющий поперечную структуру моды $\mathrm{TE}_{10}$. На входе в ЗС волноводная мода преобразуется в замедленную поверхностную электромагнитную волну, на выходе - вновь преобразуется в моду $\mathrm{TE}_{10}$ (см. [12]) Такая конфигурация соответствует условиям эксперимента. При моделировании эффективная проводимость медного слоя выбиралась равной $\sigma=2.75 \cdot 10^{7} \mathrm{~S} / \mathrm{m}$, что позволяет достичь хорошего соответствия между экспериментальными и численными результатами.

\section{4. Моделирование выходных характеристик ЛБВ}

Рассчитанные выше электродинамические параметры ЗС были использованы для моделирования выходных характеристик ЛБВ-усилителя. Расчеты проводились с использованием известных нелинейных уравнений одномерной теории ЛБВ (см., например, $[15,18])$. Уравнения движения электронов записываются в виде

$$
-\frac{d^{2} \theta}{d \xi^{2}}=\left[\left(1+C \frac{d \theta}{d \xi}\right)^{2}-\frac{v_{0}^{2}}{c^{2}}\right]^{3 / 2} \operatorname{Re}\left(F \exp (i \theta)+F_{s c}\right)
$$

где $\theta=\omega t-\beta_{e} z-$ фаза электрона, $\omega-$ частота сигнала, $\beta_{e}=\omega / v_{0}, v_{0}-$ скорость электронного пучка, $\xi=\beta_{e} C z$ - безразмерная координата, $C=\sqrt[3]{I_{0} R_{C} / 4 V_{0}}$ - параметр усиления Пирса, $I_{0}-$ постоянный ток электронного пучка, $V_{0}-$ ускоряющее напряжение, $R_{C}-$ сопротивление связи, $F=\frac{E}{2 \beta_{e} V_{0} C^{2}} \exp \left(-i \beta_{e} z\right)-$ безразмерная комплексная амплитуда волны в $3 \mathrm{C}, F_{s c}-$ безразмерная амплитуда поля пространственного заряда, которая определяется выражением

$$
F_{s c}=i q \sum_{k=1}^{N_{h}} \frac{D_{k}^{2} I_{k}}{k} \exp (i k \theta)
$$

Здесь $I_{k}=\frac{1}{\pi} \int_{0}^{2 \pi} \exp (-i k \theta) d \theta_{0}-$ безразмерные комплексные амплитуды гармоник тока, $\theta_{0}$ - начальные фазы электронов, $N_{h}$ - число учитываемых гармоник поля пространственного заряда, $q=\omega_{p}^{2} /(\omega C)^{2}$ - параметр пространственного заряда, $\omega_{p}-$ плазменная частота, $D$ - коэффициент депрессии, для которого используем выражение

$$
D^{2}=1-\frac{\sinh \left(\gamma h_{b}\right) \cosh \left[\gamma h_{b}\left(a / h_{b}-1\right)\right]}{\gamma h_{b} \cosh (\gamma a)},
$$

справедливое в случае ленточного пучка, движущегося между двумя проводящими плоскостями [15]. В формуле $(2) \quad \gamma=(\omega / c) \sqrt{\left(c / v_{0}\right)^{2}-1}, h_{b}-$ полутолщина электронного пучка, $a$ - расстояние от поверхности ЗС до середины пучка.

Безразмерная комплексная амплитуда поля подчиняется уравнению возбуждения

$$
\frac{d F}{d \xi}+i r F=-(1+b C)^{2} \frac{2 \gamma_{0}^{2}}{1+\gamma_{0}} I_{1}
$$

где $r=b-i d, b=\left(\beta-\beta_{e}\right) /\left(\beta_{e} C\right)-$ параметр несинхронности, $\beta$ - постоянная распространения волны в системе без электронного пучка, $d$ - параметр холодных потерь, $\gamma_{0}=\left(1-\left(v_{0} / c\right)^{2}\right)^{-1 / 2}-$ релятивистский масс-фактор Лоренца.

Считается, что электронный поток на входе в систему не модулирован ни по скорости, ни по плотности. Поэтому введенные выше уравнения (1), (3) необходимо дополнить граничными условиями для электронов и безразмерных амплитуд поля, которые имеют вид

$$
\theta(\xi=0)=\theta_{0},\left.\quad \frac{d \theta}{d \xi}\right|_{\xi=0}=0
$$

причем начальные фазы $\theta_{0}$ равномерно распределены на интервале $[0,2 \pi)$. Также необходимо задать граничное условие для поля

$$
F(\xi=0)=F_{m},
$$

где $F_{m}$ - постоянная амплитуда входного сигнала.

Приведенные выше уравнения решаются при помощи традиционного для СВЧ-электроники метода крупных частиц [18]. 

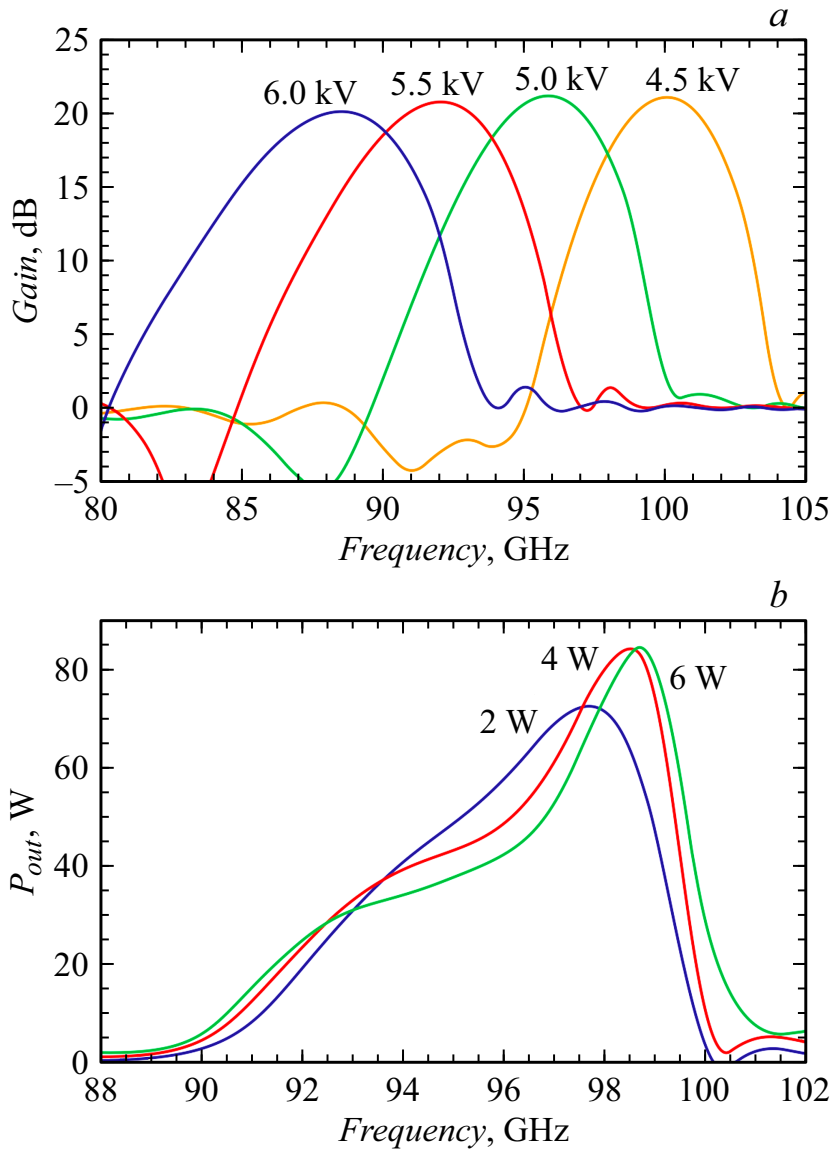

Рис. 5. Выходные характеристики ЛБВ с планарной ЗС на диэлектрической подложке длиной $1 \mathrm{~cm}$ при токе пучка $100 \mathrm{~mA}$ : $a-$ коэффициент усиления при различных напряжениях пучка; $b-$ зависимости выходной мощности от частоты при напряжении $5 \mathrm{kV}$ и различных значениях входной мощности.

Для расчета усиления в режиме малого сигнала также использовался линеаризованный вариант уравнений (1)

$$
\frac{d^{2} I_{1}}{d \xi^{2}}+\frac{q D_{1}^{2}}{\gamma_{0}^{3}} I_{1}=i \frac{F}{\gamma_{0}^{3}} .
$$

Соответственно граничные условия (4) примут вид

$$
I_{1}(\xi=0)=\left.\frac{d I_{1}}{d \xi}\right|_{\xi=0}=0 .
$$

Расчеты проводились при помощи программы, которая ранее использовалась для моделирования ЛБВ с ленточным пучком и ЗС типа плоской гребенки [15]. Как показано в [15], результаты, полученные с помощью данной программы, достаточно хорошо согласуются с современными универсальными 3D-программными пакетами CST Particle Studio и KARAT.

При моделировании длина системы была выбрана равной $1 \mathrm{~cm}$, что соответствует 77 периодам 3С. Ток пучка составлял $100 \mathrm{~mA}$. На рис. 5, а показаны зависимости коэффициента усиления в линейном режиме от частоты при различных напряжениях пучка. Видно, что усиление может превышать $20 \mathrm{~dB}$. Поскольку 3С обладает сильной дисперсией, полоса усиления оказывается достаточно узкой $(3-5 \mathrm{GHz}$ по уровню $-3 \mathrm{~dB})$. Однако имеется возможность перестройки полосы усиления с помощью изменения ускоряющего напряжения (ср. [11]). Рис. 5, b иллюстрирует нелинейные характеристики усилителя. На нем представлены зависимости выходной мощности $P_{\text {out }}$ от частоты при различных значениях мощности входного сигнала $P_{\text {in }}$ и напряжении $5 \mathrm{kV}$. С ростом $P_{\text {in }}$ частота, на которой достигается максимальная выходная мощность, смещается вправо. Насыщение происходит при входной мощности 4-6 W в окрестности частоты $99 \mathrm{GHz}$, при этом выходная мощность составляет примерно $80 \mathrm{~W}$, а электронный КПД соответственно около $15 \%$.

Отметим, что при токе пучка порядка $100 \mathrm{~mA}$ средняя плотность тока составляет примерно $465 \mathrm{~A} / \mathrm{cm}^{2}$. Хотя это значение является весьма высоким, имеются успешные примеры фокусировки и транспортировки ленточных пучков с такой плотностью тока на расстояния порядка нескольких сантиметров $[19,20]$. В данном слу-
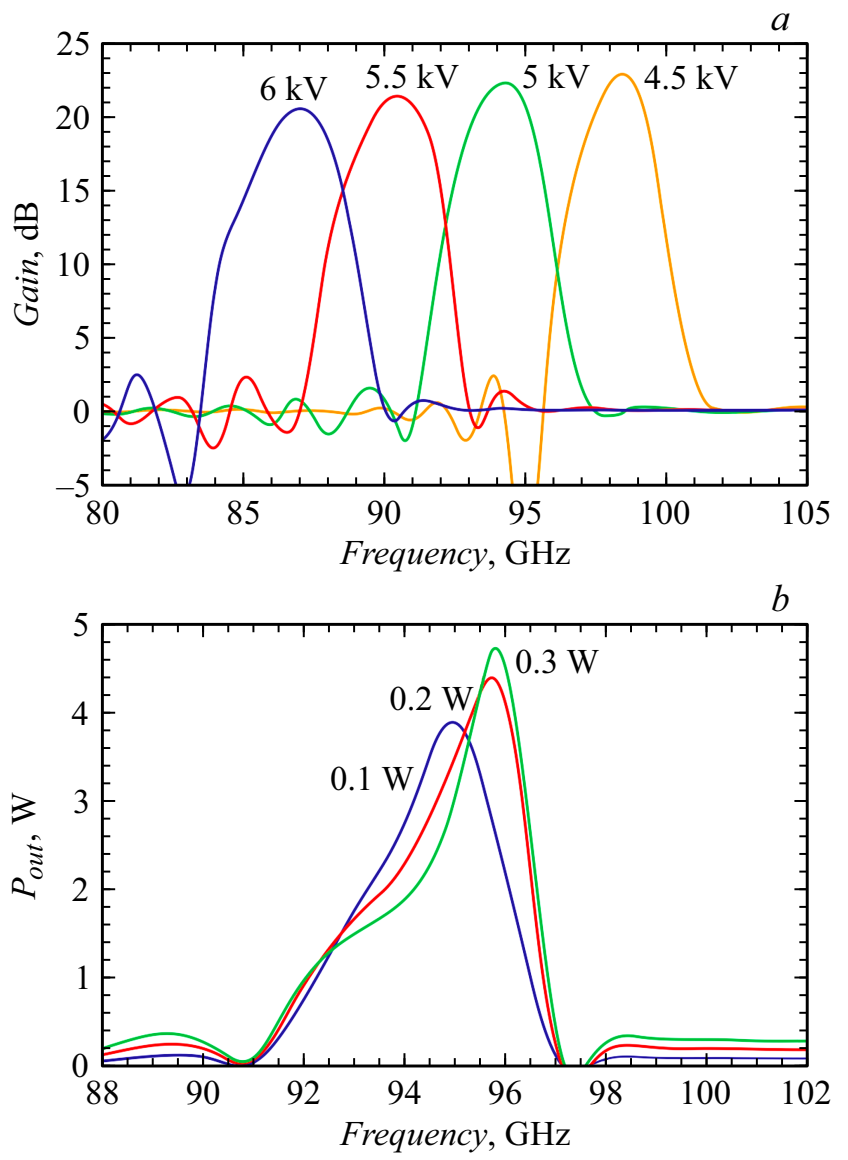

Рис. 6. Выходные характеристики ЛБВ с планарной ЗС на диэлектрической подложке длиной $2 \mathrm{~cm}$ при токе пучка $10 \mathrm{~mA}$ : $a$ - коэффициент усиления при различных напряжениях пучка; $b-$ зависимости выходной мощности от частоты при напряжении $5 \mathrm{kV}$ и различных значениях входной мощности. 
чае, ввиду большого коэффициента замедления планарной 3С, длина пространства взаимодействия составляет $1 \mathrm{~cm}$, что значительно меньше, чем для ЛБВ с ЗС типа гребенки, которые рассматривались в $[15,19,20](4 \mathrm{~cm})$. Таким образом, характерный эффект закручивания краев ленточного пучка в магнитном поле, который может привести к оседанию на поверхность ЗС и подложки, будет выражен в менышей степени.

Отметим, что недавно с помощью электронных пушек с холодной полевой эмиссией были экспериментально реализованы ленточные пучки толщиной $100-150 \mu \mathrm{m}$ и током 5-10 mA [21,22]. Поэтому представляет интерес моделирование выходных характеристик ЛБВ при токе пучка $10 \mathrm{~mA}$. Длина системы при этом была увеличена до $2 \mathrm{~cm}$ (155 периодов 3С). Результаты моделирования приведены на рис. 6. На рис. 6, $a$ представлены частотные зависимости коэффициента усиления при различных значениях ускоряющего напряжения. В данном случае в линейном режиме коэффициент усиления достигает значений 20-23 dB, однако полоса усиления оказывается несколько меньше, чем в предыдущем случае $(2-3 \mathrm{GHz})$. На рис. $6, b$ представлены зависимости выходной мощности от частоты при напряжении $5 \mathrm{kV}$ и различных значениях входной мощности. Максимальная мощность $P_{\text {out }}$ достигается при значениях входной мощности $150-300 \mathrm{~mW}$ и составляет $4-5 \mathrm{~W}$. Насыщение в данном случае происходит в окрестности частоты $96 \mathrm{GHz}$.

\section{Заключение}

В работе представлены результаты исследования планарной ЗС типа меандр на диэлектрической подложке из кварца в $W$-диапазоне $(70-110 \mathrm{GHz})$. Проведено компьютерное моделирование в программном пакете COMSOL Multiphysics, которое позволило определить основные электродинамические параметры 3С. Она обладает большим замедлением, вследствие чего напряжение синхронизма в рабочем диапазоне частот лежит в пределах $4-6 \mathrm{kV}$.

Представлена новая технология изготовления подобных электродинамических структур, основанная на методе магнетронного напыления и методе лазерной абляции. Преимуществами данной технологии является ее низкая стоимость, высокая скорость производства и технологическая гибкость. Изготовлены экспериментальные образцы ЗС и проведено исследование их $S$-параметров с помощью векторного анализатора цепей. Также проведено моделирование $S$-параметров для полноразмерной модели 3С. Численные и экспериментальные результаты хорошо согласуются друг с другом.

На основе полученных результатов проведено численное моделирование выходных характеристик ЛБВ с ленточным электронным пучком. При токе пучка $100 \mathrm{~mA}$ и длине $3 \mathrm{C} 1 \mathrm{~cm}$ коэффициент усиления в линейном режиме может превышать $20 \mathrm{~dB}$, а выходная мощность в режиме насыщения достигает $80 \mathrm{~W}$. Хотя ввиду сильной дисперсии ЗС полоса усиления достаточно узкая, имеется возможность ее перестройки при изменении ускоряющего напряжения. Также рассмотрено усиление в ЛБВ при токе пучка $10 \mathrm{~mA}$, который может быть получен в электронной пушке с полевой эмиссией. При увеличении длины $3 \mathrm{C}$ до $2 \mathrm{~cm}$ коэффициент усиления также превышает $20 \mathrm{~dB}$, а выходная мощность достигает $5 \mathrm{~W}$.

Полученные результаты позволяют утверждать, что разработанная и исследованная ЗС перспективна для использования в миниатюрных низковольтных ЛБВ $W$-диапазона средней мощности.

\section{Финансирование работы}

Работа выполнена при финансовой поддержке гранта Российского научного фонда (проект № 17-12-01160).

\section{Конфликт интересов}

Авторы заявляют, что у них нет конфликта интересов.

\section{Список литературы}

[1] Srivastava V. // J. Phys. Conf. Ser. 2008. Vol. 114. N 1. P. 012015. DOI 10.1088/1742-6596/114/1/012015

[2] Booske J.H., Dobbs R.J., Joye C.D., Kory C.L., Neil G.R., Park G.S., Park J.H., Temkin R.J. // IEEE Tr. Thz Sci. Tech. 2011. Vol. 1. N 1. P. 54-75. DOI: $10.1109 /$ TTHZ.2011.2151610

[3] Гуляев Ю.В., Жбанов А.И., Захарченко Ю.Ф., Нефедов И.С., Синицын Н.И., Торгашов Г.В. // Радиотехника и электроника. 1994. Т. 39. № 12. С. 2049-2058.

[4] Ulisse G., Krozer V. // IEEE Electr. Device Lett. 2017. Vol. 38. N 1. P. 126-129. DOI: 10.1109/LED.2016.2627602

[5] Shen F., Wei Y.-Y., Yin H.-R., Gong Y.-B., Xu X., Wang Sh., Wang W.-X., Feng J.-J. // IEEE Tr. Plasma Sci. 2012. Vol. 40. N 2. P. 463-469. DOI: 10.1109/TPS.2011.2175252

[6] Sumathy M., Augustin D., Datta S.K., Christie L., Kumar L. // IEEE Tr. Electr. Devices 2013. Vol. 60. N 5. P. 1769-1775. DOI: 10.1109/TED.2013.2252179

[7] Bai N., Feng C., Liu Y., Fan H., Shen C., Sun X. // IEEE Tr. Electr. Devices 2017. Vol. 64. N 7. P. 2949-2954. DOI: 10.1109/TED.2017.2706368

[8] Galdetskiy A., Rakova E. // Proc. of the 8th IEEE Int. Vacuum Electron. Conf. (IVEC 2017). London, UK, 2017. DOI: 10.1109/IVEC.2017.8289680

[9] Wang S., Aditya S., Xia X., Ali Z., Miao J. // IEEE Tr. Electr. Device. 2018. Vol. 65. N 6. P. 2142-2148. DOI: $10.1109 /$ TED.2018.2798575

[10] Бенедик А.И., Рожнёв А.Г., Рыскин Н.М., Синицын Н.И., Торгашов Г.В., Шалаев П.Д. // Радиотехника. 2016. № 7. C. $47-51$.

[11] Benedik A.I., Rozhnev A.G., Ryskin N.M., Sinitsyn N.I., Torgashov G.V., Torgashov R.A. // Proc. of the 18th IEEE Int. Vacuum Electron. Conf. (IVEC 2017). London, UK, 2017. DOI: 10.1109/IVEC.2017.8289499 
[12] Ryskin N.M., Rozhnev A.G., Starodubov A.V., Serdobintsev A.A., Pavlov A.M., Benedik A.I., Torgashov R.A., Torgashov G.V., Sinitsyn N.I. // IEEE Electr. Device Lett. 2018. Vol. 39. N 5. P. 757-760.

DOI: 10.1109/LED.2018.2821770

[13] Электронный ресурс. Режим доступа: https://www.comsol.ru/comsol-multiphysics

[14] Силин Р.А. Периодические волноводы. М.: Фазис, 2002. $438 \mathrm{c}$.

[15] Karetnikova T.A., Rozhnev A.G., Ryskin N.M., Fedotov A.E., Mishakin S.V., Ginsburg N.S. // IEEE Tr. Electr. Device. 2018. Vol. 65. N 6. P. 2129-2134.

DOI: 10.1109/TED.2017.2787960

[16] Starodubov A.V., Serdobintsev A.A., Pavlov A.M., Galushka V.V., Mitin D.M., Ryskin N.M. // Proc. of the 2018 IEEE Int. Vacuum Electron. Conf. (IVEC). Monterey, CA, 2018. P. 333-334. DOI: 10.1109/IVEC.2018.8391512

[17] Starodubov A.V., Serdobintsev A.A., Pavlov A.M., Galushka V.V., Ryabukho P.V., Ryskin N.M. // Proc. of the 2018 Progress in Electromagnetics Research Symposium (PIERS-Toyama). Toyama, Japan, 2018. P. 506-509. DOI: 10.23919/PIERS.2018.8597953

[18] Кач, А.М., Ильина Е.М., Манькин И.А. Нелинейные явления в СВЧ приборах О-типа с длительным взаимодействием. М.: Сов. радио, 1975. 296 с.

[19] Baig A., Gamzina D., Kimura T., Atkinson J., Domier C., Popovic B., Himes L., Barchfeld R., Field M., Luhmann N.C. // IEEE Tr. Electr. Device 2017. Vol. 64. N 5. P. 2390-2397. DOI: 10.1109/TED.2017.2682159.

[20] Field M., Kimura T., Atkinson J., Gamzina D., Luhmann N.C., Stockwell B., Grant T.J., Griffith Z., Borwick R., Hillman C., Brar B., Reed T., Rodwell M., Shin Y.-M., Barnett L.R., Baig A., Popovic B., Domier C., Barchfield R., Zhao J., Higgins J.A., Goren Y. // IEEE Tr. Electr. Device. 2018. Vol. 65. N 6. P. 2122-2128. DOI: 10.1109/TED.2018.2790411

[21] Burtsev A.A. // Electron. Lett. 2018. Vol. 54. N 13. P. 839-840. DOI: 10.1049/el.2018.0922

[22] Буриев А.А., Павлов А.А., Кицюк Е.П., Григорьев Ю.А., Данилушкин А.В., Шумихин К.В. // Письма в ЖТФ. 2017. Т. 43. Вып. 11. С. 88-94. DOI: $0.21883 /$ PJTF.2017.11.44701.16570 [Burtsev A.A., Pavlov A.A., Kitsyuk E.P., Grigor'ev Yu.A., Danilushkin A.V., Shumikhin K.V. // Tech. Phys. Lett. 2017. Vol. 43. N 11. P. 542-544. DOI: 10.1134/S1063785017060062] 